\title{
Daniela FINOCCHI (a cura di), Lingua madre duemiladiciotto - Racconti di donne straniere in Italia
}

Edizioni SEB27, Torino, 2018

Michela Marocco

(2) OpenEdition

Journals

Edizione digitale

URL: https://journals.openedition.org/esp/4818

DOI: 10.4000/esp.4818

ISSN: 2532-0319

\section{Editore}

Centre d'Information sur l'Éducation Bilingue et Plurilingue

\section{Edizione cartacea}

Data di pubblicazione: 1 décembre 2019

Paginazione: 97-103

ISSN: 1127-266X

Notizia bibliografica digitale

Michela Marocco, «Daniela FINOCCHI (a cura di), Lingua madre duemiladiciotto - Racconti di donne straniere in Italia», Éducation et sociétés plurilingues [Online], 47 | 2019, Messo online il 01 septembre 2021, consultato il 01 septembre 2021. URL: http://journals.openedition.org/esp/4818 ; DOI: https:// doi.org/10.4000/esp.4818 


\section{DANIELA FINOCGHI (A GURA DI), LINGUA MADRE DUEMILADICIOTTO - RACCONTI DI DONNE STRANIERE IN ITALIA, EDIZIONI SEB27, TORINO, 2018.}

\section{Michela Marocco}

La moitié des migrants à l'échelle mondiale sont des femmes; en Italie, les immigrées sont $54 \%$ des adultes étrangers. Les voyages des femmes sont silencieux, longs, tourmentés, heureux, dramatiques mais en tout cas silencieux. C'est le type du voyage raconté dans Lingua Madre Duemiladiciotto - Racconti di donne straniere in Italia [Langue Mère Deux mille dix-huit - Récits de femmes étrangères en Italie], une anthologie de récits de la XIIIème édition du Concours littéraire national Lingua Madre, un projet permanant de la Région Piémont adressé aux femmes étrangères (ou d'origine étrangère) résidant en Italie, avec une section réservée aux femmes italiennes qui voudraient parler des femmes étrangères qu'elles ont connues, rencontrées et qui auraient su leur transmettre «d'autres identités».

Mots-clés: femmes étrangères, immigrées, voyages, Concours Langue Mère, autres identités

On a world scale, half the migrants are women; in Italy, immigrant women make up 54\% of foreigners over 18. Women's journeys are silent, long, tormented, joyful, dramatic, but at any rate silent. That is the sort of journey recounted in Lingua Madre Duemiladiciotto - Racconti di donne straniere in Italia [Mother Tongue Two thousand eighteen - Tales of foreign women in Italy], an anthology of stories from the XIIIth edition of the national literary competition Lingua Madre, an on-going project of the Piedmont Region open to foreign women (or women of foreign descent) living in Italy, with a section for Italian women wishing to talk about the foreign women they have known and who were able to transmit "other identities".

Key-words: foreign women, immigrant women, journeys, Lingua Madre competition, other identities

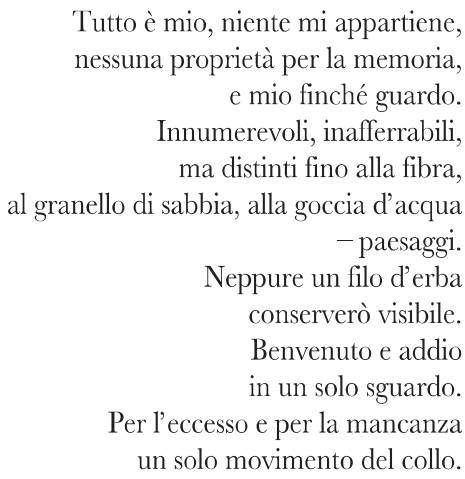




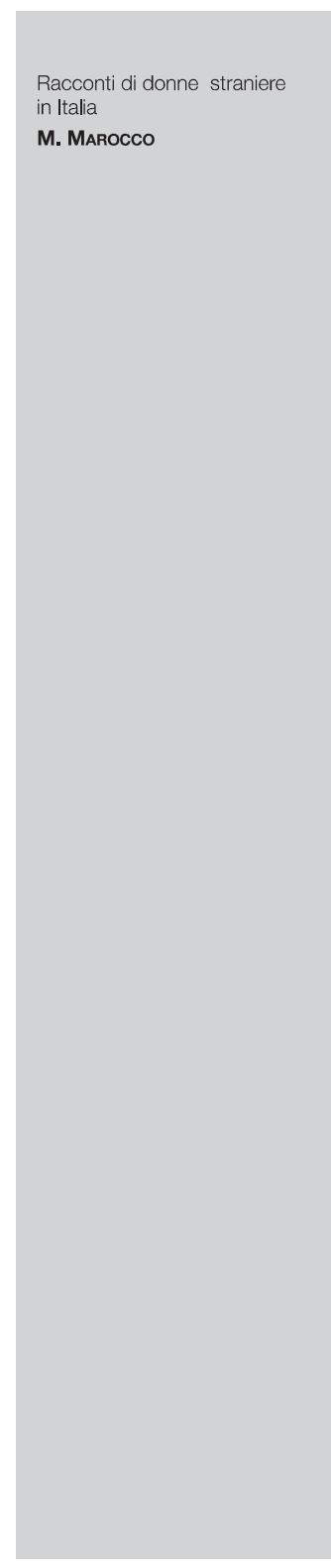

Così Wisława Szymborska, Premio Nobel per la Letteratura nel 1996, concludeva la sua "Elegia di viaggio", versi toccanti e disincantati che sembrano racchiudere in se stessi la natura profonda del viaggiare, quel muoversi da un luogo ad un altro lasciandosi spesso alle spalle tutto quello che si è conosciuto e che pian piano comincia a svanire, in favore di una nuova vita. Perché il viaggio, quello profondo e sconvolgente, quello che cambia ed evolve, è un viaggio di sola andata.

Di questo tipo di partenze le donne sono sempre più spesso principali testimoni e protagoniste benché la letteratura ne dimentichi nomi e imprese (la metà dei migranti a livello mondiale sono donne e in Italia le immigrate rappresentano il 54\% degli adulti stranieri).

Forse perché i viaggi delle donne sono silenziosi, lunghi, travagliati, felici e drammatici ma pur sempre silenziosi. Iniziano nell'anonimato di una quotidianità divenuta ormai insostenibile e si concludono in una terra straniera forti della sola speranza per un futuro diverso, per se stesse, per i propri cari. Un futuro per nulla regalato ma che bisogna conquistarsi duramente e con tenacia benché ormai gli orrori della vita precedente siano lontani.

Questo tipo di viaggio può assumere mille forme e mille volti, adattandosi ai panni della protagonista che lo intraprende, alle volte corazza, alle volte sudario, ma sempre unico e importante, decisivo. Questo è il tipo di viaggio raccontato nell'antologia Lingua Madre Duemiladiciollo - Racconli di donne straniere in Italia, edita da SEB27, volume che raccoglie i racconti della XIII edizione del Concorso letterario nazionale Lingua Madre, progetto permanente della Regione Piemonte diretto alle donne straniere (o di origine straniera) residenti in Italia, con una sezione per le donne italiane che vogliano raccontare le donne straniere che hanno conosciuto, incontrato e che abbiano saputo trasmettere loro "altre identità".

Nato nel 2005 da un'idea di Daniela Finocchi, il Concorso Lingua Madre ha raccolto dalla sua istituzione oltre 7000 autrici che da tutta Italia hanno scritto o fotografato, condiviso, svelato, confidato in questi quattordici anni di lavoro intorno alla narrazione, alla cultura, alla relazione tra donne e se a queste si aggiungono quelle che durante tutto l'anno scrivono e interagiscono con il progetto online e offline i numeri si moltiplicano. Non vengono messi limiti, né barriere, si può scrivere e fotografare a qualsiasi età e in qualsiasi condizione, che si sia una bambina delle elementari o una donna detenuta e si può partecipare da sole, con opere realizzate a quattro mani, ma anche in gruppo. 


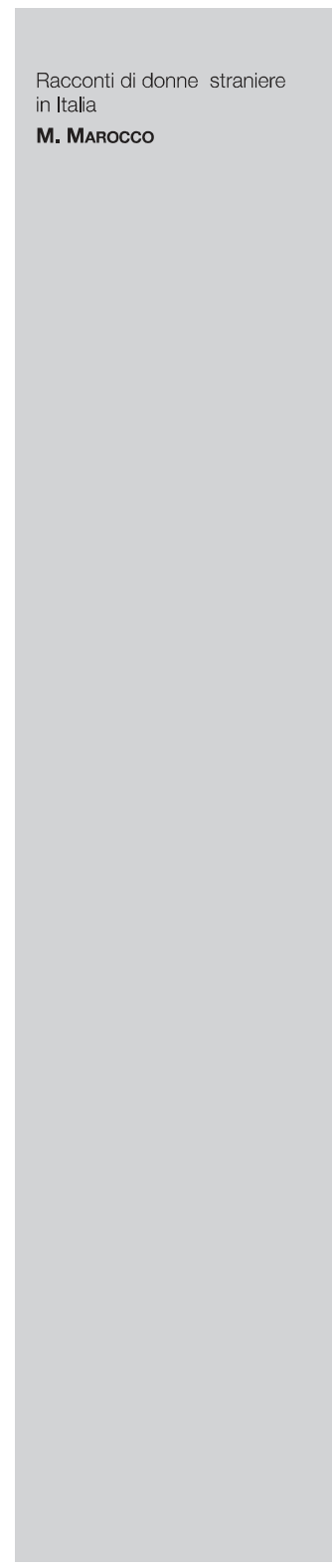

Scopo del progetto è quindi dare voce a chi spesso non ce l'ha e creare occasioni di scambio, relazione, conoscenza.

Ogni anno i racconti selezionati per la pubblicazione vengono raccolti in un'antologia che quasi organicamente sembra costruirsi attorno ad un tema, un invisibile filo conduttore che collega tutte le storie, come tante variazioni di un unico punto di vista.

Lingua Madre Duemiladiciotto sembra quindi costituirsi attorno a partenze e arrivi, famiglie che si ricongiungono o si spezzano per sempre, movimenti di crescita e conoscenza di se stesse e degli altri, navicelle spaziali, vortici temporali, ricordi.

Viaggi che donano, viaggi che tolgono, viaggi che arricchiscono coloro che li vivono, trasportando queste donne nella versione futura di se stesse.

Un viaggio a piedi scalzi attraverso il deserto con il solo obiettivo di una vita migliore. Un viaggio alla ricerca delle proprie radici nella figura di un nonno mai conosciuto, caduto in guerra. Un viaggio per passare le vacanze in un Paese lontano, in quella casa delle origini che ora raccoglie ricordi e malinconia. Un viaggio nel tempo dal sapore fantastico e romantico, un viaggio spaziale in un altro mondo così terribilmente simile al nostro. I viaggi raccolti in questa antologia si declinano in tantissime variazione diverse ma sempre costituiscono un momento fondamentale nella vita di colei che li racconta, delineando quasi una nuova geografia, basata sull'emozione e sul ricordo, sul coraggio e sulla speranza.

I viaggi rappresentati diventano percorsi di formazione, che non solo muovono le loro protagoniste nello spazio, ma danno loro anche gli strumenti per capire e per capirsi, per mettersi in relazione, per affrontare un mondo con loro spesso troppo crudele. Dimostrazione ne sono i racconti vincitori raccolti nell'antologia. In "Ricordi rubati" di Dunja Badnjević, l'anziana protagonista serba arriva a compiere azioni impensabili dopo essere stata vittima di quella violenza fisica e psicologica che solo gli espatriati conoscono: "Sentii la mia testa girare. Come stessi morendo. Qui, davanti alla mia casa, con degli estranei che mi negavano anche la memoria. Che uccidevano anche il ricordo. Che mi privavano della mia vita".

Attraverso la scrittura le donne imparano a dare corpo e senso al silenzio, facendone spazio di comunicazione e possibilità. Uno spazio che, come scrive Daniela Finocchi, il Concorso Lingua Madre vuole tener vivo e valorizzare, dando voce - proprio tramite le pause, le interruzioni e i ritmi della scrittura - a tutte le donne, di ogni paese e latitudine, e offrendo loro un luogo auten- 


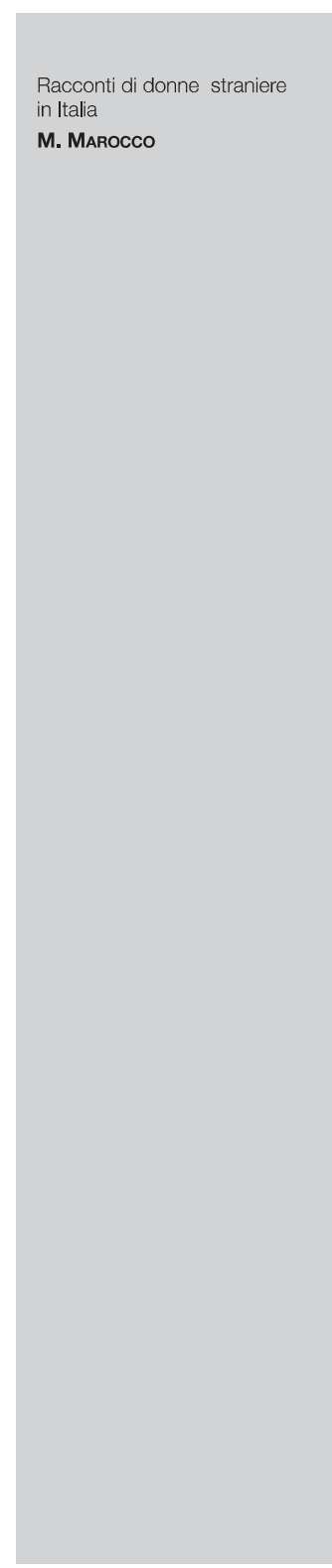

tico di espressione e rappresentazione di sé, della realtà e del contesto in cui vivono. Ecco quindi che "il silenzio da assenza si fa etere in cui trasmettere sentimenti di amore, dipendenza, relazione, umanità e cura. Tutti valori di cui le donne sono portatrici e che diventano, come ricorda Elena Pulcini, quasi schermo della disuguaglianza".

In questo modo, i commossi ricordi quotidiani che Melita Ferkovic racconta in "Parole sospese sulla neve" riescono a esprimere tutta la dolcezza e la malinconia di chi parte ma non dimentica. Sotto una bianca coltre affiorano memorie giovanili di quel paese che un tempo era casa, i momenti di crescita, le cicatrici, il rapporto con la madre c con esso il riconoscimento della genealogia femminile quale necessità vitale. "Sono sempre in ansia per quello che trovo dietro la porta quando mia madre mi apre, dopo tanto tempo che non la vedo - scrive Ferkovic - Trattengo il respiro suonando il campanello. La sua figura minuta è ogni volta più piegata, il passo lento, ma più diventa piccola di statura, più io avverto qualcosa di grande attorno a lei: c'è una luce, nella sua pazienza salda e nel suo sguardo in attesa".

In "K.19" di Valeria Rubino vengono raccontate le storie delle ospiti di un centro di accoglienza, con il freddo, agghiacciante distacco di istantanee fotografiche. Sono tantissimi i ricordi che Rubino propone, veri e propri ritratti che si modellano negli occhi di chi legge grazie ad una narrazione spietata e inclemente, che non addolcisce nulla ma solamente mostra. Ritratti costruiti sull'emozione e non sulla fisicità: "W. è eritrea e ha 22 anni. Nel suo Paese era un soldato. Durante l'addestramento ha dovuto subire un'iniezione "per non rimanere incinta". Da allora non le viene la mestruazione. Questo tipo di pratica viene applicata per evitare di perdere soldati per colpa delle gravidanze, sia che si tratti di una donna che ha avuto un rapporto consensuale sia che si parli di una violenza sessuale. Cose che succedono".

Al contrario, Marcela Luque in "Hind dappertutto" narra la strabiliante, inaspettata eppure così comune amicizia fra due donne di origini assai diverse, sottolineando la fondamentale importanza di quella rete femminile che nasce nelle difficoltà. Una rete personificata dalla protagonista, Hind, dea ex machina sempre presente, che viene osservata e raccontata da una voce narrante grata e ricca di dolcezza per questa amica fuori dal comune. Infatti, come scrive Luque, "non parlavano mai di quanto fosse dura tirare avanti, di come le loro piatte giornate fossero piene di mille difficoltà, di tutti i sogni rimasti nei loro rispettivi cassetti e che non avrebbero mai potuto realizzare, di quanto si sarebbero sentite 


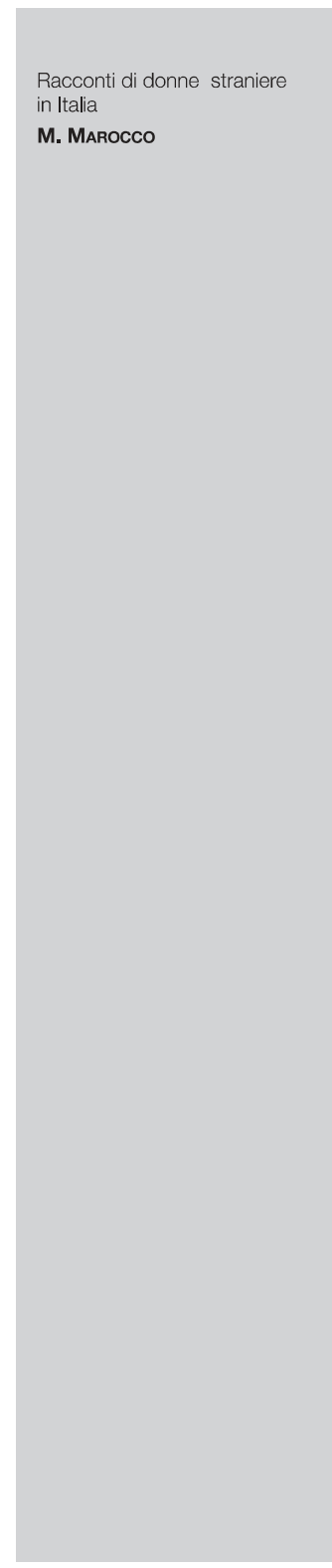

così sole se non si fossero tenute l'una all'altra. Perché non c'era bisogno di parlarne, entrambe ci stavano passando, entrambe lo sapevano ed entrambe comprendevano esattamente quello che l'altra stava attraversando. $\mathrm{E}$ in quell'intimità raggiunta di un'intensità tale che non c'era bisogno di dire certe cose, soprattutto lì, c'era Hind".

In "Parole perdute" Dorota Czalbowska si concentra sul complicato mondo delle adozioni internazionali riportando la voce straziante eppure piena d'amore di un padre in rapporto con il figlio straniero. Attraverso la difficoltà di comunicazione in questa famiglia, la Czalbowska tratteggia dinamiche familiari complesse e profonde, in cui l'amore per un figlio deve confrontarsi con una fiducia difficile da guadagnare. "Ogni nostro tentativo di intavolare un discorso che avesse un minimo di senso, aveva come unico scopo quello di essere compresi da te - scrive Czalbowska - Ci scrutavi come se stessi facendo un calcolo di probabilità della riuscita dell'operazione, misuravi il grado di fiducia da concederci e parlavi con occhi attenti ai quali nulla sfuggiva, nemmeno la più impercettibile smorfia. A volte accennavi un sorriso. Eravamo avvolti da un uragano emotivo in grado di spazzare via un intero Paese, tanto che mentre cercavamo di risultare disinvolti, i nostri nervi erano tesi come corde di violino".

Infine, è con il racconto "Per aspera ad astra", vincitore del Primo Premio, che il tema del viaggio irrompe prepotentemente e si riappropria di tutti quei significati letterali e metaforici espressi dagli altri, inglobandoli in qualche modo tutti, diventando simbolo e rappresentazione di tutta la raccolta. Aicha Fuamba, insieme alla docente Sofia Teresa Bisi, racconta con voce quasi monotonale, infantile il terribile viaggio che dalla Repubblica Democratica del Congo l'ha portata in Italia: un penoso e a tratti insopportabile cammino, per i/le protagonisti/e ma anche per i/le lettori/trici, che culminerà in tragedia. Un viaggio che oppone meschine dinamiche familiari a momenti in cui proprio da queste dinamiche scaturisce il più profondo e puro sentimento d'amore. "Le onde sono sempre più alte, impediscono di vedere intorno; ma vicino ci sono gli scogli: un impatto forte, terribile causa uno squarcio sulla fiancata dell'imbarcazione. Trattengo il fiato, ho il cuore in gola, mi sento viva e quindi mi metto a pregare: "Dio, ti prego, fa' che non succeda più..." La barca incontra un'onda enorme, tutto cambia prospettiva in un attimo: siamo catapultati in mare. È tutto un gridare, agitarsi, aggrapparsi a qualunque cosa. Chi si cerca, chi nuota, chi resta immobile: molti di noi non sanno nuotare e sopportano il loro atroce destino. Io 


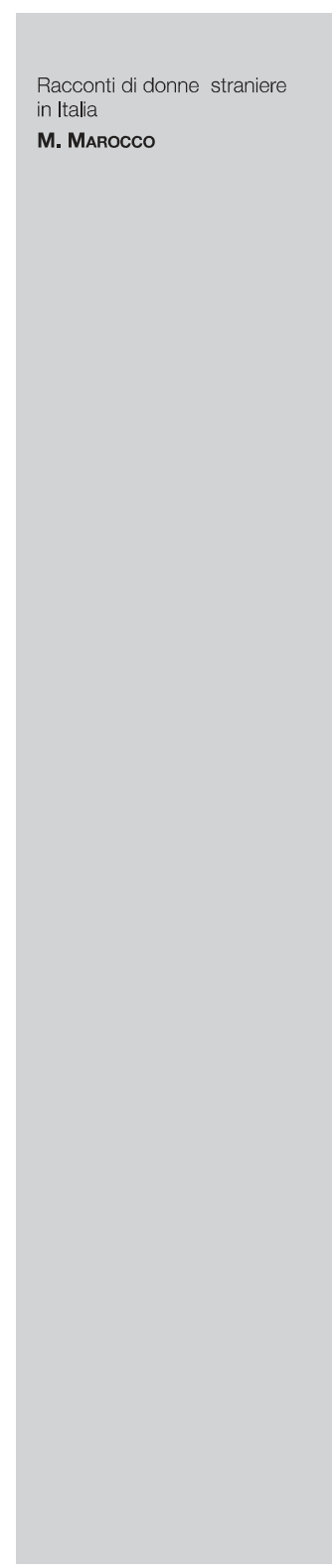

ho un salvagente che papà ha comprato prima di partire; all'improvviso sento che qualcuno me lo vuole strappare. Per reazione decido di afferrarlo con più forza: non so nuotare". Così Fuamba descrive il terribile epilogo di un viaggio travagliato e molto doloroso, a cui sua madre non è sopravvissuta.

Sono ancora moltissime le storie che si susseguono nel volume, esplorando stili e linguaggi differenti.

Per queste donne il viaggio è l'unica soluzione possibile, non scelta ma necessità fisica ed emotiva. Che sia una fuga o una breve visita, che sia inventato o atrocemente vero, il viaggio compiuto da queste protagoniste si prospetta come l'unica via percorribile, per cambiare, per conoscere, per sopravvivere.

Ma il viaggio oltre che movimento nello spazio è anche e soprattutto movimento all'interno di sé, un movimento di introspezione che insegna sempre qualcosa di nuovo a chi lo compie.

Come accade per Elyria, la protagonista raccontata da Catherine Lacey in Nessuno scompare davvero - che da un giorno all'altro, dopo la perdita della sorella, lascia la sua apparente vita perfetta per andare dall'altra parte del mondo - così ci sono autrici il cui viaggio nasce da un lutto, dalla perdita di qualcuno o di qualcosa. In questi casi la partenza si fa necessità intrinseca, componente dell'io più profondo, per mettersi in gioco, per interrogarsi su ogni aspetto dell'esistenza. Così il viaggio, quello fisico e reale, diventa metafora per quello che è in verità un viaggio all'interno della mente delle stesse narratrici.

L'antologia Lingua Madre Duemiladiciotto è ricca di viaggi introspettivi che prendendo l'avvio da situazioni reali o metaforiche aiutano le protagoniste a raggiungere una nuova e diversa coscienza di sé, più profonda, più vera. Così possiamo leggere di viaggi alla scoperta (o riscoperta) delle proprie origini, viaggi indietro nel tempo sull'onda di ricordi scatenati da innocenti farfalle o dalla musica di un pianoforte lontano, oppure ancora viaggi all'interno della propria lingua, sia quella materna sia quella d'adozione, alla ricerca di un'identità sfuggente.

Queste donne travalicano ogni sorta di confine e che sia fisico o immateriale poco importa, perché in questo passaggio avviene sempre un arricchimento, della protagonista e del lettore con lei. Le autrici superano difficoltà, oltrepassano mari e valicano montagne, portando in un nuovo mondo qualcosa di quello vecchio, portando nella nuova vita qualcosa di quella vecchia.

I confini vengono abbattuti in queste storie che, attraverso esperienze estremamente peculiari e personali, profilano realtà comuni a tutti/e e in cui tutti possiamo immedesimarci. Amicizie 


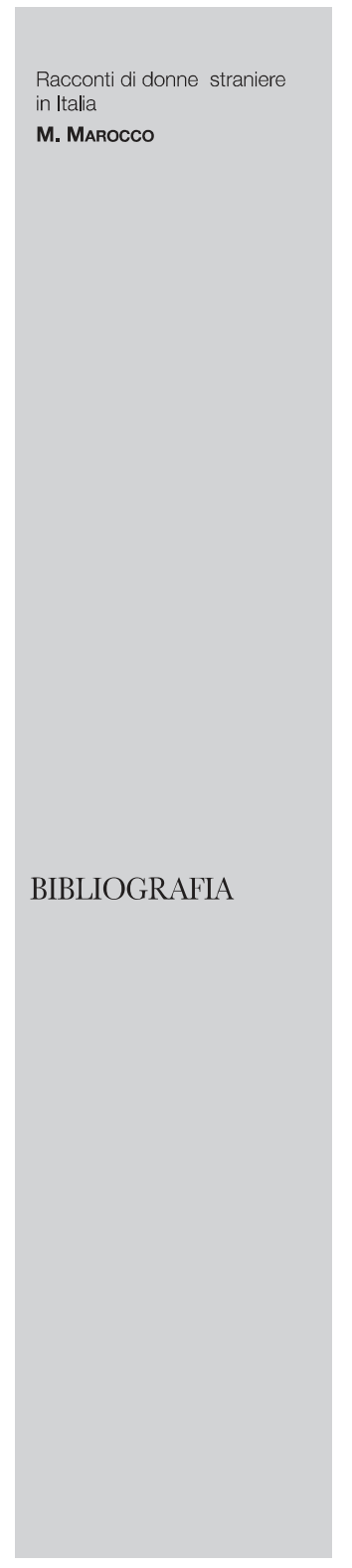

improbabili che diventano ancore di salvataggio per donne agli opposti eppure così simili, ragazze che incontrano il primo amore, madri che lottano per la salvezza dei loro figli.

Dalle pagine prendono vita innumerevoli personaggi: arruolate per missioni su pianeti lontani o trasportate in reami magici attraverso antichi armadi, eroine di una fiaba macabra dalle tinte fin troppo reali o alla ricerca di un contatto con una madre distante, in fuga da famiglie troppo conservatrici o alle prese con una gravidanza difficile.

Le donne che vengono rappresentate sono forti, caparbie, fantasiose, dolci ma soprattutto testimoniano la necessità imprescindibile dell'apertura verso l'altro/a, immaginando mondi in cui la differenza unisce invece di separare.

Sono donne che fanno proprio il quindicesimo consiglio di Chimamanda Ngozi Adichie all'amica neomamma per crescere una figlia femminista (in Cara Ijeawele): "Insegnale la differenza. Rendi la differenza naturale, rendila normale. Insegnale a non attribuire un valore particolare alla differenza. E il motivo per farlo non è essere giusti o carini, ma semplicemente umani e pratici. Perché la differenza è la realtà del nostro mondo. E insegnandole la differenza, le fornisci gli strumenti per sopravvivere in questo mondo così vario".

FINOCGHI D. 2014. Geo-grafie del silenzio, Mimesis Edizioni, Milano

LACEY C. 2016. Nessuno scompare davvero, BigSur, Roma.

NGOZI A. CHIMAMANDA. 2017. Cara Ijeawele, Einaudi, Torino.

SZYMBORSKA W. 2009. La gioia di scrivere - Tutte le poesie (19452009), Adelphi, Milano. 